CLINICAL STUDY

\title{
Evaluation of an enzyme immunoassay for plasma-free metanephrines in the diagnosis of catecholamine-secreting tumors
}

\author{
Michel Procopiou*, Hazel Finney ${ }^{1}$, Scott A Akker, Shern L Chew, William M Drake, Jacky Burrin ${ }^{1}$ \\ and Ashley B Grossman \\ Department of Endocrinology, St Bartholomew's Hospital, West Smithfield, London EC1A 7BE, UK and ${ }^{1}$ Department of Clinical Biochemistry, \\ Royal London Hospital, 4th Floor Pathology and Pharmacy Building, 80 Newark Street, London E1 2ES, UK \\ (Correspondence should be addressed to A B Grossman; Email: a.b.grossman@qmul.ac.uk) \\ *(M Procopiou is now at Hôpital de la Providence, Faubourg de l'Hôpital 81, CH-2001 Neuchâtel, Switzerland)
}

\begin{abstract}
Objective: To define the test characteristics of an enzyme immunoassay (EIA) for plasma-free metanephrines (metanephrine and normetanephrine) in the diagnosis of pheochromocytoma and paraganglioma.

Design: Prospective observational design from a single University Hospital. Twenty-four hour urine for catecholamines and plasma for free metanephrines were collected from patients with a clinical suspicion of pheochromocytoma or paraganglioma. Patient records were reviewed for clinical data, follow-up, imaging and laboratory results to establish or exclude the diagnosis of pheochromocytoma. Patients and methods: Out of 178 consecutive patients, 10 had a paraganglioma and 12 had a pheochromocytoma: 156 were finally judged not to harbour active tumors and were therefore considered as controls. The main outcome measure was the diagnosis or exclusion of paraganglioma or pheochromocytoma and test characteristics of plasma-free metanephrines measured by EIA.

Results: Urinary epinephrine had a sensitivity of $45.5 \%$ and norepinephrine a sensitivity of $75 \%$ (98.8\% specificity) for the diagnosis of pheochromocytoma. Plasma-free metanephrine and normetanephrine both had a sensitivity of $66.7 \%$ and a specificity of $100 \%$, but when combined (either positive) they demonstrated a $91.7 \%$ sensitivity with a preserved specificity of $100 \%$. For the diagnosis of paraganglioma, urinary norepinephrine gave slightly better results than plasma-free metanephrines, but combined testing was of no additional value.

Conclusions: Plasma-free metanephrines measured by EIA have better diagnostic test characteristics than urinary catecholamines in the diagnosis of pheochromocytoma. The EIA offers a simple and effective measurement of plasma-free metanephrines.
\end{abstract}

European Journal of Endocrinology 161 131-140

\section{Introduction}

Pheochromocytomas are rare neuroendocrine tumors arising from chromaffin cells of the adrenal medulla or extra-adrenal paraganglia. Tumors of extra-adrenal origin are now usually referred to as paragangliomas (1). Different tests are available to the clinician for their biochemical diagnosis: they are based on plasma or urinary measurement of the direct secretory products of the adrenomedullary-sympathetic system or their metabolites, specifically catecholamines or their metanephrine derivatives (2). In general, as the catecholamines epinephrine and norepinephrine may be secreted in an intermittent fashion, most authorities now tend to recommend assessment of their metabolites metanephrine and normetanephrine as these provide a more robust measure of catecholamine output (3). According to the recommendations for the diagnosis of pheochromocytoma from the First International Symposium on Pheochromocytoma, the initial assessment of secretory capacity should include the measurements of fractionated metanephrines in urine or plasma, or both, as available (4). However, plasma-free metanephrines are currently analyzed by cumbersome and technically demanding techniques such as HPLC, or HPLC coupled with mass spectrometry, which limit their availability. We have therefore conducted a prospective study to define the test characteristics of a new enzyme immunoassay (EIA) for plasma-free metanephrines in the diagnosis of pheochromocytoma and paraganglioma in a cohort of patients from a single referral centre. 


\section{Patients and methods}

\section{Patients}

Consecutive patients attending the Endocrine Clinic at St Bartholomew's Hospital (London, UK) for suspicion of catecholamine-secreting tumors were included in this study, which was agreed by the Institutional Review Board. The diagnosis of pheochromocytoma was suspected on clinical grounds (classical or suspicious symptoms, hypertension that was severe, resistant to multiple medications, or seen at a young age, etc.) or because of the incidental finding of an adrenal mass. A number of patients were tested as part of a surveillance program in the context of genetic syndromes. In the great majority of patients, the diagnosis was excluded on normal biochemistry, an absence of abnormal imaging, and a failure to progress on regular review. All patients included in the study were tested by undergoing a 24-h urinary collection for the measurement of catecholamines (epinephrine, norepinephrine, and dopamine), with a single fasting blood test for plasma-free metanephrines (metanephrine and normetanephrine) analysis by EIA. Specific dietary recommendations are not routinely given to our patients as catecholamines and metanephrines are not materially affected by dietary intake. No interrogation regarding paracetamol was undertaken as this agent, which may contaminate results, is included in a whole variety of remedies of which most patients are unaware, so specific questioning is of dubious value.

The clinician in charge of the patient was free to decide whether imaging was necessary (adrenal magnetic resonance imaging (MRI) or computed tomography (CT), plus radionuclide imaging with ${ }^{123} \mathrm{I}$-meta-iodo benzylguanidine $\left({ }^{123} \mathrm{I}-\mathrm{MIBG}\right)$ and the form of follow-up required. All clinicians were blinded to the plasma metanephrine results. At the end of the recruitment period, one of the authors (M P), blinded to the plasma metanephrine results, reviewed all patient records for the following information: working diagnosis made by the attending physician, follow-up results, imaging studies, and potential explanations for false positive urinary catecholamines results (such as obstructive sleep apnoea, medications, etc). This final diagnosis was concordant with the diagnosis made by the attending clinician in all cases, with the exception of a single patient. Following this analysis, a single patient with positive plasma metanephrines, initially considered not to have a pheochromocytoma, was reviewed and reclassified as showing a true pheochromocytoma (see Results).

A secretory pheochromocytoma or paraganglioma was diagnosed if the urinary catecholamines were positive ( $>99$ th centile) with positive adrenal or extra-adrenal imaging. The diagnosis was confirmed histologically in all patients subjected to operative removal. In the case of paragangliomas, the diagnosis of a non-secreting tumor was made in the presence of typical radiological findings with normal urinary catecholamines results and positive surgical pathology. The diagnosis of a pheochromocytoma was considered to be excluded if biochemical testing was negative in patients with a low clinical probability, and no further evidence appeared during prolonged follow-up at a mean follow-up of 1.5 years ( $538 \pm 210$ days).

Where appropriate, negative cross-sectional imaging

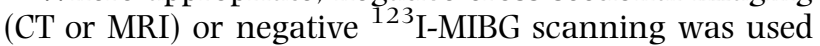
as corroborative evidence. If the 24-h urinary catecholamines were positive ( $>99$ th centile), negative adrenal, abdominal, and, in some cases, thoracic, cross-sectional imaging, plus ${ }^{123}$ I-MIBG scanning, were utilized to exclude the diagnosis. When the diagnosis was rejected in spite of positive urinary catecholamines, an alternate explanation for the false positive results was sought and obtained.

\section{Urinary catecholamines analysis by HPLC with electrochemical detection}

Twenty-four hour urinary specimens were collected from patients into acid $(6 \mathrm{M} \mathrm{HCl} ; \mathrm{pH}<3)$ bottles. No dietary restrictions were enforced (as noted above). Measurement of urinary catecholamines (norepinephrine, epinephrine, and dopamine) was performed by HPLC-electrochemical detection (ECD) following a technique previously described in detail (5).

\section{Plasma-free metanephrines analysis by EIA}

Plasma-free metanephrine and normetanephrine were determined using an EIA commercial kit manufactured by Labor Diagnostica Nord GmbH, Nordhorn, Germany, and supplied by Immunodiagnostic Systems Ltd (IDS), Tyne and Wear, UK.

Extraction Metanephrine and normetanephrine in calibrators, controls, and patient samples were precipitated following $60 \mathrm{~min}$ incubation at room temperature in tubes in which two precipitants were added then vortex mixed. Following centrifugation at $3000 \boldsymbol{g}$ for $10 \mathrm{~min}, 100 \mu \mathrm{l}$ of supernatant was used for the following acylation step.

Acylation For each supernatant, $100 \mu \mathrm{l}$ was pipetted into the respective wells of the acylation plate. Addition of $25 \mu \mathrm{l}$ of buffer, followed by $25 \mu \mathrm{l}$ of freshly diluted acylation reagent was added to all wells. The acylation plate was then incubated for $15 \mathrm{~min}$ at room temperature on an orbital shaker $(14 \boldsymbol{g})$.

EIA From each acylated supernatant, $50 \mu \mathrm{l}$ was used in the metanephrine assay and $10 \mu \mathrm{l}$ was used in the normetanephrine assay. Each supernatant volume was pipetted into the respective wells of each of the metanephrine and normetanephrine EIA plates, followed by $100 \mu \mathrm{l}$ of each respective antiserum. 
Each plate was covered with foil and left to incubate at $2-8{ }^{\circ} \mathrm{C}$ for $>40 \mathrm{~h}$. After this incubation time, each plate was washed and blotted three times before $100 \mu \mathrm{l}$ of enzyme conjugate was added and incubated for $30 \mathrm{~min}$ at room temperature on an orbital shaker. There was further washing and blotting followed by the addition of $100 \mu \mathrm{l}$ of substrate to all wells with incubation at room temperature for $\sim 20 \mathrm{~min}$. The final step was to add $100 \mu \mathrm{l}$ of stop solution to all wells followed by absorbance reading of the solution using a microplate reader at $450 \mathrm{~nm}$ with reference wavelength between 620 and $650 \mathrm{~nm}$. The absorbance reading took place within the $10 \mathrm{~min}$ allowed from the time the stop solution was added.

Assay sensitivity was $10 \mathrm{pg} / \mathrm{ml}$ for both plasma metanephrine and normetanephrine. Inter-assay coefficients of variation were $8.8 \%$ at $34 \mathrm{pg} / \mathrm{ml}$ and $9.4 \%$ at $339 \mathrm{pg} / \mathrm{ml}$ for plasma metanephrine, and $14.5 \%$ at $62 \mathrm{pg} / \mathrm{ml}$ and $8.55 \%$ at $625 \mathrm{pg} / \mathrm{ml}$ for plasma normetanephrine respectively. Quality control samples were added to each assay run $(n=10)$ in order to compute the inter-assay coefficient of variation.

\section{Institutional and manufacturers' normal limits for biochemical assays}

The normative limits for 24-h urinary catecholamines were $<144 \mathrm{nmol} / 24 \mathrm{~h}$ for epinephrine, $<560 \mathrm{nmol} / 24 \mathrm{~h}$ for norepinephrine, and $<3194 \mathrm{nmol} / 24 \mathrm{~h}$ for dopamine: these were based on a previous assessment of the 95th centile (5). According to the manufacturer's recommendations, normal values for plasma-free metanephrines are $<90 \mathrm{pg} / \mathrm{ml}$ for metanephrine and $<200 \mathrm{pg} / \mathrm{ml}$ for normetanephrine.

\section{Statistical analysis}

Figures with receiver-operating characteristic curves (ROC curves) and ROC curve calculations (area under ROC curve, 95\% confidence interval, CI) were done with MedCalc (version 9.6.4.0). All other figures were drawn and calculations (mean, 95\% CI, sensitivities, and specificities) were performed using GraphPad Prism 5 (GraphPad Software Inc., La Jolla, CA 92037, USA).

\section{Results}

The total cohort consisted of 236 samples. Out of these, 30 were taken from the same patients $<12$ months apart and were excluded from the analysis. In another 22 cases, urinary catecholamines were not available, thus leaving 184 separate samples for the analysis. These 184 samples came from 178 different patients (mean age 45.2 years, 95\% CI, \pm 18 years, 108 females and 70 males). Six patients were tested twice (more than 12 months apart). Of the total group, 156 patients
(161 samples) were finally judged not to harbour active tumors and were therefore considered as controls. Out of the 22 patients (23 samples) with catecholaminesecreting tumors, 10 were paragangliomas (Table 1) (one patient with two samples, only one sample was used for all calculations) and 12 were pheochromocytomas (Table 1). The final diagnosis was made after a mean follow-up of 1.5 years $(538 \pm 210$ days).

In the 178 subjects, the reasons for testing were clinical symptoms or investigation for secondary hypertension in $78(43.8 \%)$, a confirmed or suspected genetic syndrome in 55 (30.9\%), follow-up after treatment for pheochromocytoma or paraganglioma in $23(12.9 \%)$, and investigations after the discovery of an adrenal incidentaloma in 22 (12.4\%). The genetic syndromes consisted of 15 patients with multiple endocrine neoplasia (MEN; three MEN1, nine MEN2A, and three MEN2B), 13 von Hippel-Lindau (VHL) patients, 4 patients with succinate dehydrogenase (SDH) mutations, 2 patients with neurofibromatosis type 1 , and 5 patients with a positive family history for familial paragangliomas. In the 16 remaining subjects, a genetic syndrome was suspected in terms of the clinical background (ten MEN2, four VHL, and two SDH mutations).

\section{Twenty-four hour urinary catecholamines}

A total of 184 results from 178 different patients were available for urinary catecholamine analysis. The detailed results are given in Table 2 and displayed graphically in Fig. 1. In the 156 controls, the mean $24 \mathrm{~h}$ urinary epinephrine was $34.1 \mathrm{nmol} / 24 \mathrm{~h}$, norepinephrine was $308.8 \mathrm{nmol} / 24 \mathrm{~h}$, and dopamine was $1741 \mathrm{nmol} / 24 \mathrm{~h}$. For the 12 pheochromocytomas, the mean 24 h urinary epinephrine was $786 \mathrm{nmol} / 24 \mathrm{~h}$, norepinephrine $2407 \mathrm{nmol} / 24 \mathrm{~h}$, and dopamine $2970 \mathrm{nmol} / 24 \mathrm{~h}$. For one of the patients with a pheochromocytoma, the urinary epinephrine result was not available because of analytical interference probably due to a medication. For the ten paragangliomas, the mean $24 \mathrm{~h}$ urinary epinephrine was $36.5 \mathrm{nmol} / 24 \mathrm{~h}$, norepinephrine was $1947 \mathrm{nmol} / 24 \mathrm{~h}$, and dopamine $4358 \mathrm{nmol} / 24 \mathrm{~h}$.

Using the institutional cut-offs (abnormal $24 \mathrm{~h}$ urinary catecholamines if $>144 \mathrm{nmol} / 24 \mathrm{~h}$ for epinephrine, if $>560 \mathrm{nmol} / 24 \mathrm{~h}$ for norepinephrine, and if $>3194 \mathrm{nmol} / 24 \mathrm{~h}$ for dopamine), the sensitivities (specificities) were $45.5 \%(98.8 \%)$ for epinephrine, $75.0 \%(91.3 \%)$ for norepinephrine, and $16.7 \%$ $(93.8 \%)$ for dopamine in the diagnosis of pheochromocytoma. In the diagnosis of paraganglioma, sensitivities (specificities) were 0\% (98.8\%) for epinephrine, $70.0 \%(91.3 \%)$ for norepinephrine, and $40 \%(93.8 \%)$ for dopamine. The confidence intervals for sensitivities and specificities, and ROC curves, are given in Table 3. 


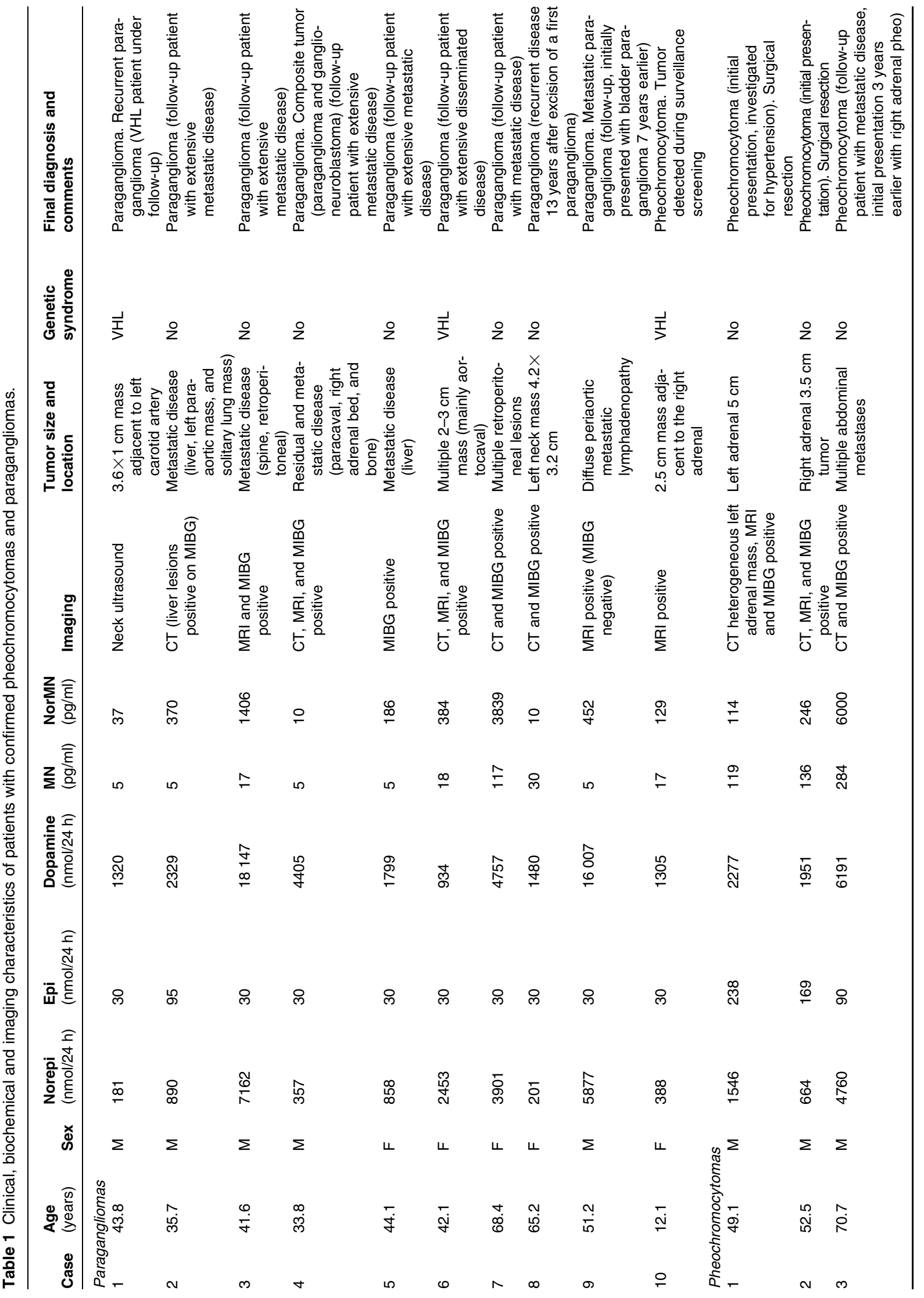




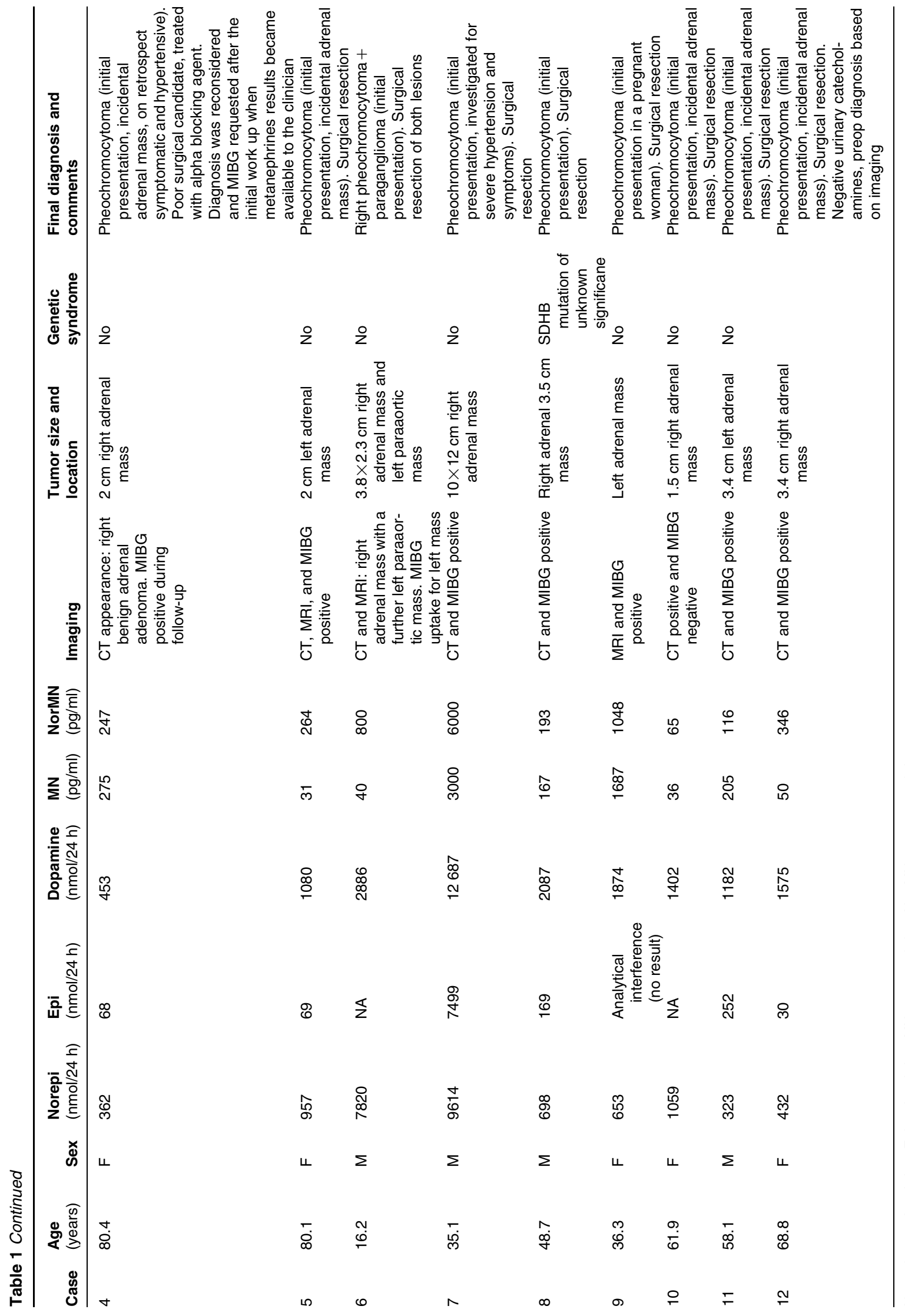


Table 2 Twenty-four hour urinary catecholamines and plasma-free metanephrines: mean values (lower and upper $95 \% \mathrm{Cl}$ of the mean).

\begin{tabular}{lccc}
\hline & Pheochromocytoma $(n=12)$ & Paragangliomas $(n=10)$ & Controls $(n=161)$ \\
\hline 24-h urinary catecholamines $(\mathrm{nmol} / 24-\mathrm{h})$ & & & \\
Epinephrine & $785.8^{\mathrm{a}}(-711.0-2283)$ & $36.5(21.8-51.2)$ & $34.1(30.6-37.6)$ \\
Norepinephrine & $2407(371.8-4443)$ & $1947(330.3-3564)$ & $308.8(284.5-333.1)$ \\
Dopamine & $2970(821.7-5119)$ & $4358(609.9-8106)$ & $1741(1559-1924)$ \\
Plasma-free metanephrines $(\mathrm{pg} / \mathrm{ml})$ & $502.5(74.8-1080)$ & $24.1(0.06-48.3)$ & $17.6(15.2-20.0)$ \\
Metanephrine & $1287(124.5-2698)$ & $646.9(208.8-1503)$ & $43.1(36.9-49.2)$ \\
$\quad$ Normetanephrine & & &
\end{tabular}

${ }^{a} n=11$ for 24-h epinephrine (see text).

\section{Plasma-free metanephrines}

In the same cohort of 178 patients, 184 results were available for plasma metanephrines analysis. The detailed results are given in Table 2 and displayed graphically in Fig. 1. In the 156 controls, the mean plasma metanephrine was $17.6 \mathrm{pg} / \mathrm{ml}$ and mean plasma normetanephrine $43.1 \mathrm{pg} / \mathrm{ml}$. In our control population, the highest value for metanephrine was 74 and $180 \mathrm{pg} / \mathrm{ml}$ for normetanephrine. For the 12 pheochromocytomas, mean plasma metanephrine was $502.5 \mathrm{pg} / \mathrm{ml}$ and mean plasma normetanephrine was $1287 \mathrm{pg} / \mathrm{ml}$. For the ten paragangliomas, mean plasma metanephrine was $24.1 \mathrm{pg} / \mathrm{ml}$ and mean plasma normetanephrine was $646.9 \mathrm{pg} / \mathrm{ml}$. Using the manufacturer's cut-offs (abnormal plasma metanephrine if $>90 \mathrm{pg} / \mathrm{ml}$; abnormal normetanephrine if $>200 \mathrm{pg} / \mathrm{ml})$, the sensitivity $(66.7 \%)$ and specificity $(100 \%)$ were identical for metanephrine and for normetanephrine in the diagnosis of pheochromocytoma. In the diagnosis of paraganglioma, sensitivities (specificities) were 10\% (100\%) for metanephrine and $40 \%(100 \%)$ for normetanephrine. For the diagnosis of pheochromocytoma, ROC curves for 24-h urinary norepinephrine, plasma metanephrine, plasma normetanephrine, sum of plasma metanephrines, and sum of normalized ratios of plasma metanephrines are given in Fig. 2.

\section{Test characteristics for combined urinary epinephrine and norepinephrine versus combined metanephrine and normetanephrine (for pheochromocytoma only)}

We then assessed whether combining the results from the urinary epinephrine and norepinephrine, and plasma-free metanephrine and normetanephrine respectively, would change the test utility in the diagnosis of pheochromocytoma. For paragangliomas, this analysis was of no added value as urinary epinephrine was negative in all cases, and plasma-free metanephrine was positive in only a single case with a positive normetanephrine: thus, combined testing failed to improve the utility of either urinary catecholamines or plasma metanephrines.
For plasma normetanephrine and metanephrine (using the same manufacturer's cut-offs: abnormal metanephrine if $>90 \mathrm{pg} / \mathrm{ml}$; abnormal normetanephrine if $>200 \mathrm{pg} / \mathrm{ml}$ ), the combination of results (either
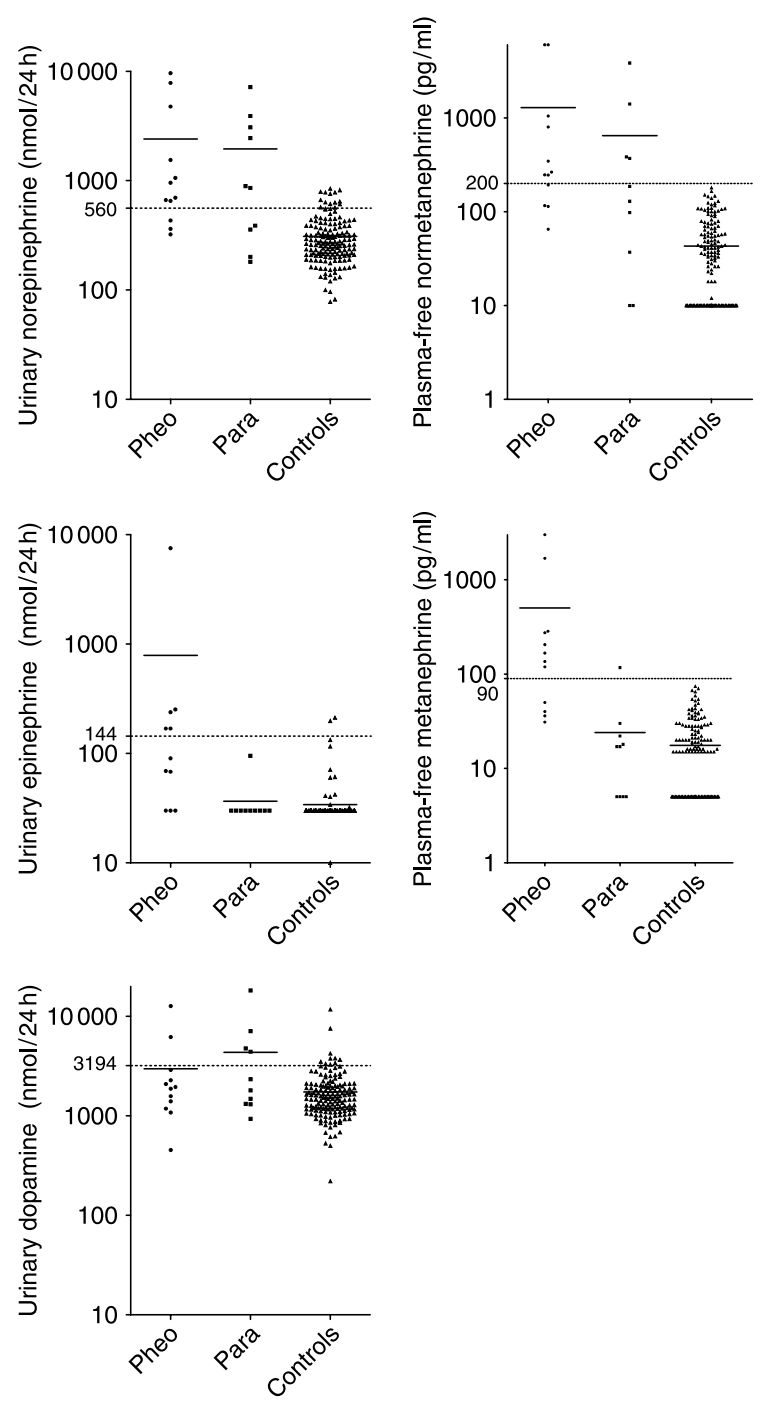

Figure 1 Test results for 24-h urinary catecholamines (norepinephrine, epinephrine, and dopamine) and plasma-free metanephrines (metanephrine and normetanephrine). Dashed lines are cut-off values. 
one to be positive) retained a $100 \%$ specificity (i.e. not a single false positive result), but sensitivity was increased to $91.7 \%$ for the diagnosis of pheochromocytoma (a single pheochromocytoma would have been be missed; Table 4 and Fig. 3). In order to construct a ROC curve with a single variable, we divided the metanephrine and normetanephrine values by their respective upper limit reference $(90 \mathrm{pg} / \mathrm{ml}$ for free plasma metanephrine and $200 \mathrm{pg} / \mathrm{ml}$ for free plasma normetanephrine). Then, we added these two ratios and obtained a single variable integrating the two free metanephrines as a sum of normalized ratios. On the ROC curve, the cut-off with the highest accuracy was 1.48 (sensitivity $91.7 \%$ and specificity $100 \%$ ). The area under the ROC curve had the highest value at 0.987 (95\% CI: 0.956-0.998) compared with the ROC curves for the single results (see Table 3). The simple sum of metanephrine and normetanephrine gives basically the same results at a cut-off of $228 \mathrm{pg} / \mathrm{ml}$, but the sum of normalized ratios allows easier comparisons with other methods if they have different reference values.

In comparison, the combination of urinary epinephrine and norepinephrine (either one to be positive above our institutional cut-offs) increased the sensitivity of urinary catecholamines to $83.3 \%$ but at the cost of a loss of specificity (90.1\%). By raising the cut-offs for epinephrine to $>220 \mathrm{nmol} / 24 \mathrm{~h}$ and $>650 \mathrm{nmol} / 24 \mathrm{~h}$ for norepinephrine, the maximal specificity obtained was $95.6 \%$ with the same sensitivity of $83.3 \%$ (Table 4 and Fig. 4).

\section{Discussion}

In this study, we investigated the utility of a new EIA for plasma-free metanephrines in comparison with our current analytical technique, urinary catecholamines measured by HPLC-ECD. Ideally, the EIA should have been tested against metanephrines measurements by HPLC or gas chromatography coupled with tandem mass spectroscopy; however, at the time we performed our study, this technique for plasma or urinary metanephrines was not routinely available in our institution, and this is still the case in many other centres worldwide. We decided to analyze separately the results for paragangliomas and pheochromocytomas because of their different secretion patterns.

Our study shows that plasma-free metanephrines measured by EIA have better test characteristics in the diagnosis of pheochromocytoma than urinary catecholamines. Plasma metanephrine showed a sensitivity of $66.7 \%$ and a specificity of $100 \%$ at a cut-off level of $90 \mathrm{pg} / \mathrm{ml}$. At a cut-off level of $200 \mathrm{pg} / \mathrm{ml}$, plasma normetanephrine had similar characteristics. The sensitivity and specificity of plasma-free metanephrine was higher than its urinary catecholamine counterpart, epinephrine. The sensitivity of urinary norepinephrine was higher than that of plasma-free 


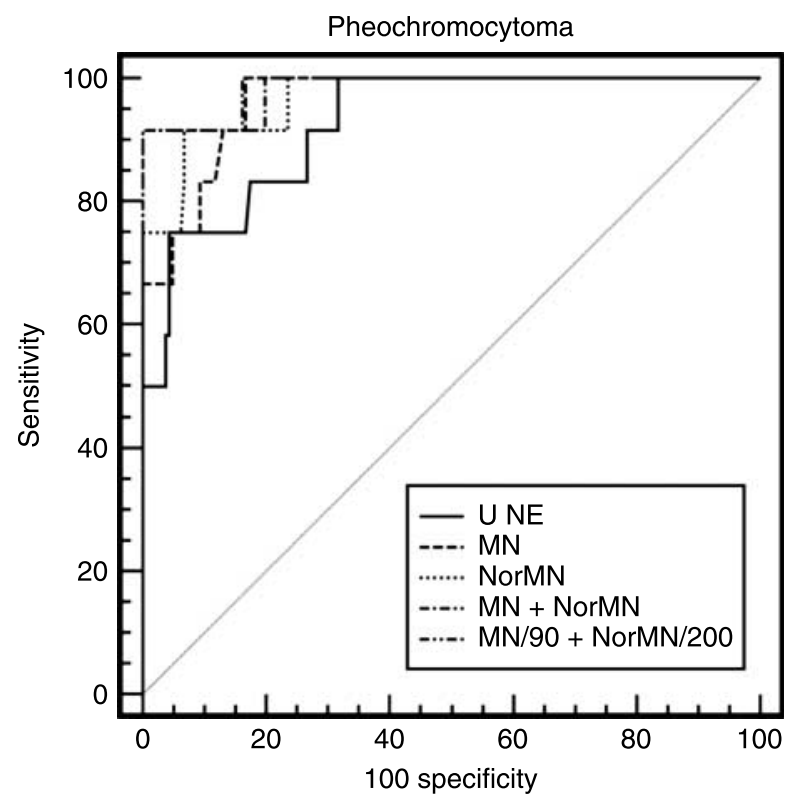

Figure 2 Multiple comparison of ROC curves in the diagnosis of pheochromocytoma. U NE, 24-h urinary norepinephrine; MN, plasma metanephrine; NorMN, plasma normetanephrine;

$\mathrm{MN}+$ NorMN, sum of plasma metanephrines; MN/90+NorMN/200, sum of normalized ratios of plasma-free metanephrines.

normetanephrine but at the price of a lower specificity. The area under the ROC curve for plasma-free normetanephrine was similar to that for urinary norepinephrine, but assessment of either free metanephrine or normetanephrine had the highest sensitivity and specificity. The combination of both metanephrines results (either metanephrine or normetanephrine to be positive) still maintained a $100 \%$ specificity (i.e. not a single false positive result) with a sensitivity of $91.7 \%$ for the diagnosis of pheochromocytoma (one pheochromocytoma would be missed). In our cohort, lowering the metanephrine cut-offs to diagnose the one missed pheochromocytoma would lead to an unacceptable loss of specificity. Combined plasma metanephrines had higher sensitivity and specificity compared with combined urinary epinephrine and norepinephrine even if catecholamine cut-offs were raised to improve their specificity.
In the diagnosis of paraganglioma, urinary norepinephrine seemed to perform slightly better than plasma-free normetanephrine with a sensitivity of $70 \%$ (40\% for plasma-free normetanephrine) and a specificity of $91.3 \%$ (100\% for plasma-free normetanephrine) and an area under ROC curve of 0.787 ( 0.778 for plasma-free normetanephrine). Paragangliomas show a different catecholamine secretory pattern compared with pheochromocytomas, a fact that has been well established for many years. Extra-adrenal pheochromocytomas - paragangliomas - rarely secrete epinephrine, and paragangliomas are generally less often secretory compared with pheochromocytomas (6).

In the recommendations for the diagnosis of pheochromocytoma from the First International Symposium on pheochromocytoma, the authors stated that 'measurements of fractionated metanephrines (i.e. normetanephrine and metanephrine measured separately) in urine or plasma provide superior diagnostic sensitivity to measurements of the parent catecholamines' (4). Catecholamines and metanephrines are excreted by the kidneys and renal function impairment leads to variations in their levels. However, in patients with renal failure, total metanephrines levels are elevated but free metanephrines are not affected (7). Drug interference can cause false positive metanephrines results. Measurements of metanephrines by mass spectrometry should minimize potential analytical interference, but this technique is not widely available as yet (8). Metanephrines can also be elevated without pheochromocytoma in some situations of major physical stress (e.g. surgery, stroke, obstructive sleep apnoea, etc.) (8).

In an international study including the National Institutes of Health (NIH), plasma-free metanephrines and urinary fractionated metanephrines had the highest sensitivities (99 and 97\% respectively) (3). The specificity was $86 \%$ for urinary catecholamines, 93\% for urinary total metanephrines, and $89 \%$ for plasmafree metanephrines, but was the lowest for urinary fractionated metanephrines at $69 \%$. Based on ROC curve analysis, plasma-free metanephrines had the best test characteristics when different cut-offs were used for sensitivity and specificity (3). In a study from the Mayo Clinic, fractionated plasma metanephrines still had the highest sensitivity (97\%) compared with a sensitivity of

Table 4 Test characteristics (sensitivity and specificity) for combined 24-h urinary epinephrine and norepinephrine versus combined plasma-free metanephrines in the diagnosis of pheochromocytoma.

Pheochromocytoma

Sensitivity \% $(95 \% \mathrm{Cl}) \quad$ Specificity $\%(95 \% \mathrm{Cl})$

24-h urinary catecholamines

24-h urinary epinephrine $>144$ and/or norepinephrine $>560 \mathrm{nmol} / 24 \mathrm{~h}$

24-h urinary epinephrine $>220$ and/or norepinephrine $>650 \mathrm{nmol} / 24 \mathrm{~h}$

$83.3 \%(51.6-97.9 \%)$

$90.1 \%(84.3-94.2 \%)$

Plasma-free metanephrines

Plasma-free metanephrine >90 and/or plasma-free normetanephrine $>200$ pg/ml 91.7\% (61.5-99.8\%) $\quad 100 \%$ (97.7-100\%) 


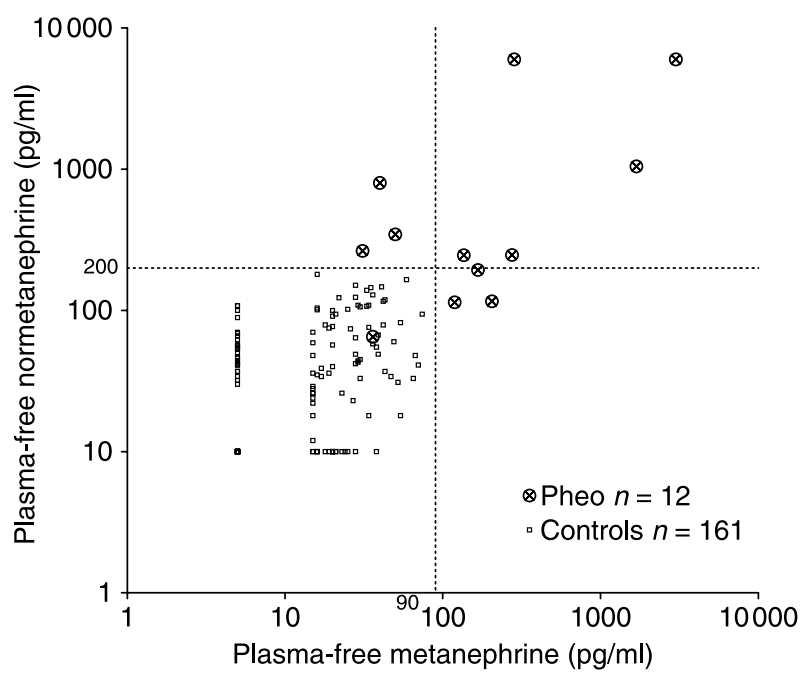

Figure 3 Test results for combined testing with plasma-free metanephrines in pheochromocytoma.

$90 \%$ for urinary total metanephrines and catecholamines (either test positive), although the difference was not statistically significant. However, the specificity of fractionated plasma metanephrines was $85 \%$ compared with $98 \%$ for the combined urinary measurements (9). The authors' conclusions were that while plasma-free metanephrines offer the most sensitive test for a pheochromocytoma, because of low specificity its routine use in screening a low probability population might give rise to too many false positive results (9). Measurement of 24-h urinary total metanephrines and

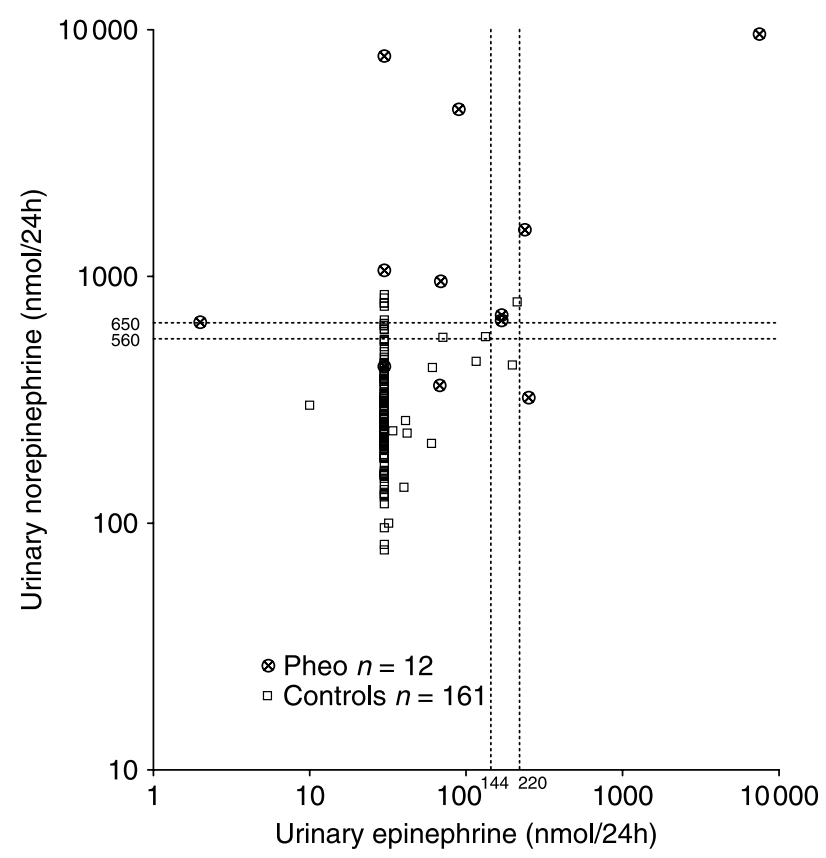

Figure 4 Test results for combined testing with 24-h urinary norepinephrine and epinephrine in pheochromocytoma. catecholamines would be more suitable for testing lowrisk subjects (the most frequent situation), whereas plasma-free metanephrines should be reserved for selected high-risk patients (such as familial endocrine syndromes) (9). Plasma-free metanephrines in both studies were analyzed using HPLC. In an editorial commenting upon the latter study, Eisenhofer emphasized that 'assays of plasma free metanephrines are (...) not as easy to establish and run on a routine basis as conventional assays of urinary metanephrines and catecholamines'. Even at the NIH, transfer of the technology from the research to the routine laboratory environment was not without some difficulty, despite the presence of highly competent and skilled technical staff' (10). Thus, while either plasma-free or urinary fractionated metanephrines were considered to be the most accurate biochemical markers of catecholaminesecreting tumors, the technical complexity of the assay(s) involved rendered their wide-scale use problematic. Testing of plasma metanephrines with an EIA may represent an alternative for centres that do not have access to the most accurate tandem mass spectrometry for plasma metanephrines, particularly in smaller units.

A more recent study from a single French centre, also using HPLC, has compared the test characteristics of fractionated metanephrine measurements (urinary and plasma) to catecholamine measurements (urinary and plasma) (11). The assay characteristics of metanephrines were always better than those of the catecholamines from which they were derived. Urinary metanephrines yielded the highest specificity and plasma metanephrines the highest sensitivity, in concordance with the conclusions of Sawka et al. $(9,11)$. In a retrospective study from Scotland, urinary free metanephrines had a sensitivity of $100 \%$ (specificity of $94 \%$ ) compared with the sensitivity of $84 \%$ for urinary catecholamines, but plasma metanephrines were not evaluated in that study (12). However, to date few studies have reported results using a technology other than HPLC to assay plasma-fractionated metanephrines. Unger and colleagues used an RIA developed by the same manufacturer as the EIA studied here (13). In their series of 24 sporadic pheochromocytomas, plasma normetanephrine was the best single parameter with a sensitivity of $91.7 \%$ and a specificity of $95.6 \%$, using a normetanephrine threshold of $>126 \mathrm{pg} / \mathrm{ml}$ derived from ROC curve analysis. We now show that a similar but simpler assay using EIA has very favorable characteristics for the investigation and diagnosis of catecholaminesecreting tumors. A recent study from China, using the same EIA as reported here, has reported that the combination of plasma-free metanephrine and normetanephrine (either positive) had a sensitivity of $96.7 \%$ and a specificity of $86.3 \%$ (14). They calculated their cut-off values as mean +2 s.D. in controls $(n=51)$, which were $91.9 \mathrm{pg} / \mathrm{ml}$ for metanephrine and $134.5 \mathrm{pg} / \mathrm{ml}$ for 
normetanephrine. However, their study had several limitations in that patients were recruited only through referral for ${ }^{131}$ I-MIBG, the diagnosis of pheochromocytoma was based exclusively on ${ }^{131}$ I-MIBG imaging, and no comparison with other biochemical tests was available in the relatively small control population.

In conclusion, our study is a prospective work conducted in a referral centre with experience in pheochromocytoma and paraganglioma management. Special care was taken in reviewing patient notes for the diagnosis of cases versus controls taking into account the clinical probability, urinary catecholamines results, cross-sectional and nuclear imaging when available, and follow-up. This study found that the diagnostic characteristics of fractionated plasma metanephrines (metanephrine and normetanephrine), as measured by EIA, are superior to the measurement of 24-h urinary catecholamines analyzed by HPLC-ECD. At the relevant cut-offs employed, the EIA for metanephrines showed a perfect specificity with a high sensitivity. While the total number of catecholamine-secreting tumors was still relatively small, we believe that our results are particularly robust regarding the specificity as we used as a control group not normal volunteers, but a patient population with a suspicion of pheochromocytoma. Thus, a positive result is almost certainly diagnostic of a catecholamine-producing tumor, either a pheochromocytoma or a paraganglioma. The absence of false positive results would be especially useful when testing a low probability population (9) where the test still provides high sensitivity, but criteria for further investigation may need to be modified where there is a high a priori suspicion of a tumor. In such cases, followup with urinary metanephrines and/or plasma chromogranin A has been suggested to be of value (15). Further evaluation of this EIA is required with a larger number of cases (pheochromocytomas and paragangliomas) for a better assessment of its sensitivity.

\section{Declaration of interest}

The authors have no conflict of interest to declare.

\section{Funding}

We are grateful to the St Bartholomew's Cancer Research Committee for financial support for this study. During his fellowship at St Bartholomew's Hospital, Dr Michel Procopiou received an unrestricted educational grant through the '3E' (Exchange in Endocrinology Expertise) fellowship program, an exchange program set up by the Section/Board of Endocrinology of the UEMS (European Union of Medical Specialists) and by Novo Nordisk A/S. Dr Michel Procopiou is indebted to Dr Eric Grouzmann and Dr Thierry Buclin (Division of Clinical Pharmacology and Toxicology, Lausanne University Medical School, Switzerland) for their comments on the combined metanephrines results.

\section{References}

1 Lenders JW, Eisenhofer G, Mannelli M \& Pacak K. Phaeochromocytoma. Lancet 2005366 665-675.

2 Grossman A, Pacak K, Sawka A, Lenders JW, Harlander D, Peaston RT, Reznek R, Sisson J \& Eisenhofer G. Biochemical diagnosis and localization of pheochromocytoma: can we reach a consensus? Annals of the New York Academy of Sciences 20061073 332-347.

3 Lenders JW, Pacak K, Walther MM, Linehan WM, Mannelli M, Friberg P, Keiser HR, Goldstein DS \& Eisenhofer G. Biochemical diagnosis of pheochromocytoma: which test is best? Journal of the American Medical Association 2002287 1427-1434.

4 Pacak K, Eisenhofer G, Ahlman H, Bornstein SR, GimenezRoqueplo AP, Grossman AB, Kimura N, Mannelli M, McNicol AM \& Tischler AS. Pheochromocytoma: recommendations for clinical practice from the First International Symposium. Nature Clinical Practice. Endocrinology and Metabolism 2007 92-102.

5 Ross GA, Newbould EC, Thomas J, Bouloux PM, Besser GM, Perrett D \& Grossman A. Plasma and 24 h-urinary catecholamine concentrations in normal and patient populations. Annals of Clinical Biochemistry 199330 38-44.

6 Erickson D, Kudva YC, Ebersold MJ, Thompson GB, Grant CS, Van Heerden JA \& Young WF Jr. Benign paragangliomas: clinical presentation and treatment outcomes in 236 patients. Journal of Clinical Endocrinology and Metabolism 200186 5210-5216.

7 Eisenhofer G. Free or total metanephrines for diagnosis of pheochromocytoma: what is the difference? Clinical Chemistry 200147 988-989.

8 Kudva YC, Sawka AM \& Young WF Jr. Clinical review 164: the laboratory diagnosis of adrenal pheochromocytoma: the Mayo Clinic experience. Journal of Clinical Endocrinology and Metabolism 200388 4533-4539.

9 Sawka AM, Jaeschke R, Singh RJ \& Young WF Jr. A comparison of biochemical tests for pheochromocytoma: measurement of fractionated plasma metanephrines compared with the combination of 24-hour urinary metanephrines and catecholamines. Journal of Clinical Endocrinology and Metabolism 2003 88 553-558.

10 Eisenhofer G. Editorial: biochemical diagnosis of pheochromocytoma - is it time to switch to plasma-free metanephrines? Journal of Clinical Endocrinology and Metabolism 200388 550-552.

11 D’Herbomez M, Forzy G, Bauters C, Tierny C, Pigny P, Carnaille B, Pattou F, Wemeau JL \& Rouaix N. An analysis of the biochemical diagnosis of 66 pheochromocytomas. European Journal of Endocrinology 2007156 569-575.

12 Boyle JG, Davidson DF, Perry CG \& Connell JM. Comparison of diagnostic accuracy of urinary free metanephrines, vanillyl mandelic acid, and catecholamines and plasma catecholamines for diagnosis of pheochromocytoma. Journal of Clinical Endocrinology and Metabolism 200792 4602-4608.

13 Unger N, Pitt C, Schmidt IL, Walz MK, Schmid KW, Philipp T, Mann K \& Petersenn S. Diagnostic value of various biochemical parameters for the diagnosis of pheochromocytoma in patients with adrenal mass. European Journal of Endocrinology 2006154 409-417.

14 Gao YC, Lu HK, Luo QY, Chen LB, Ding Y \& Zhu RS. Comparison of free plasma metanephrines enzyme immunoassay with (131)IMIBG scan in diagnosis of pheochromocytoma. Clinical and Experimental Medicine 20088 87-91.

15 Algeciras-Schimnich A, Preissner CM, Young WF Jr, Singh RJ \& Grebe SK. Plasma chromogranin A or urine fractionated metanephrines follow-up testing improves the diagnostic accuracy of plasma fractionated metanephrines for pheochromocytoma. Journal of Clinical Endocrinology and Metabolism 200893 91-95.

Received 27 March 2009

Accepted 1 April 2009 